\title{
Interest of Myocutaneous Plasty after Extended Sternal Resection
}

\author{
Ahmed Ben Ayed*, Abdessalem Hentati, Walid Abid, lyadh Ghorbel and Khalil Nouri \\ Department of Thoracic Surgery, Respiratory Medicine, Hedi Chaker University Hospital, Sfax, Tunisia
}

\begin{abstract}
Background: Thoracic wall reconstruction is commonly performed by using muscle flaps or prosthetic materials. We try through this article to show the possibility of myoplasty in extended thoracic wall resections.

Case report: A 33-year-old woman had been treated by chemo-radiotherapy, for undifferentiated carcinoma of the nasopharyngeal type. The CT-scan completed by magnetic resonance showed a $10 \mathrm{~cm}$ sternal mass centered by the manubriosternal articulation with an infiltration of left and right second sternocostal joints and intercostal muscles. The diagnosis of a single sternal metastasis of nasopharyngeal carcinoma was established.

After the resection of anterior arc of right ribs (from the first to the third), resection of internal right clavicle edge, transverse sternotomy above xiphoid process, resection of anterior arc of left ribs (from the first to the third), resection of internal left clavicle edge, sternal tumor and sternal body were removed en-bloc without a $2 \mathrm{~cm}$ residual extension which was marked by metallic clips. A myocutaneous plasty using pectoralis major and pectoralis minor muscles covered the chest wall defect after the release of the pectoralis major from its humeral attachment.
\end{abstract}

The postoperative course was uneventful. Residual tumor was treated by radiotherapy with no disease recurrence sign for 3 years later.

Conclusion: The choice of muscle to use depends on the location and the extent of the defect to be repaired. The knowledge of the anatomy of the muscles is essential to obtain a good quality flap.

Keywords: Nasopharyngeal carcinoma; Sternal resection; Chest wall reconstruction; Myocutaneous plasty

\section{Introduction}

The thoracic wall has a major static and dynamic role in respiratory function, which explains the need for efficient repair after parietal resection. Thoracic wall reconstruction is commonly performed by using muscle flaps or prosthetic materials [1,2].

Because the use of prosthetic materials can be impossible in certain situations, we try through this article to show the possibility of myoplasty in extended thoracic wall resections.

\section{Case Report}

A 33-year-old woman had been treated by chemo-radiotherapy, 2 years previously, for undifferentiated carcinoma of the nasopharyngeal type (UCNT) classified T4N1M0 with a complete remission. She was presented at our department with a presternal swelling measuring 10 $\mathrm{cm}$, diagnosed during the oncological follow-up as a single UCNT metastasis.

The CT-scan completed by magnetic resonance (Figures $1 \mathrm{~A}$ and 1B) showed a $10 \mathrm{~cm}$ sternal mass cantered by the manubriosternal articulation with an infiltration of left and right second sternocostal joints and intercostal muscles. The tumor had a contact with the aorta cross and the left brachiocephalic veins without an encroachment signs. The diagnosis of a single sternal metastasis of nasopharyngeal carcinoma (NPC) was established after a percutaneous biopsy.

Considering the age of the patient, her good performance status and the unique metastasis, the decision of a resection surgery followed by local radiotherapy was taken by a multidisciplinary meeting, including thoracic surgeon, plastic surgeon, oncologist, radiologist, and radiotherapist, to increase survival and to improve quality of life.

\section{Surgical resection}

After the resection of anterior arc of right ribs (from the first to the third), resection of internal right clavicle edge, transverse sternotomy above xiphoid process, resection of anterior arc of left ribs (from the first to the third), resection of internal left clavicle edge, a $2 \mathrm{~cm}$ residual tumor extension was discovered sheathing the phrenic nerve, encroaching the pulmonary artery trunk and the left subclavian vein. Given the major surgical risk of a complete resection, sternal tumor and sternal body were removed en-bloc without the $2 \mathrm{~cm}$ residual extension which was marked by metallic clips (Figure 2A-2C). Wide enough margins were respected to eliminate other residual malignant tissue. A large anterior thoracic wall defect exposing mediastinum and medial portions of lungs needed an immediate reconstruction to cover soft tissue, mediastinum, left and right pleural cavities, and to preserve respiration mechanical forces.

\section{Reconstruction and result}

A Myocutaneous plasty using pectoralis major and pectoralis minor muscles covered the chest wall defect after the release of the pectoralis major from its humeral attachment (Figure 3A). The postoperative course was uneventful, except an anterior paradoxical respiration with no functional impact. The patient was discharged on postoperative day 7. Three months after the operation the patient started strengthening her anterior chest wall and paradoxical respiration movements decreased (Figure 3B). Residual tumor was treated by radiotherapy with no disease recurrence sign for 3 years later.

*Corresponding author: Ahmed Ben Ayed, Department of Thoracic Surgery Respiratory Medicine, Hedi Chaker University Hospital, Sfax, Tunisia, Tel: 21623935354; E-mail: ahmed.benayed.tunisia@gmail.com

Received January 21, 2018; Accepted February 16, 2018; Published February 23, 2018

Citation: Ayed AB, Hentati A, Abid W, Ghorbel I, Nouri K (2018) Interest of Myocutaneous Plasty after Extended Sternal Resection. J Pulm Respir Med 8: 447. doi: 10.4172/2161-105X.1000447

Copyright: (๑) 2018 Ayed AB, et al. This is an open-access article distributed under the terms of the Creative Commons Attribution License, which permits unrestricted use, distribution, and reproduction in any medium, provided the original author and source are credited. 
Citation: Ayed AB, Hentati A, Abid W, Ghorbel I, Nouri K (2018) Interest of Myocutaneous Plasty after Extended Sternal Resection. J Pulm Respir Med 8: 447. doi: 10.4172/2161-105X.1000447

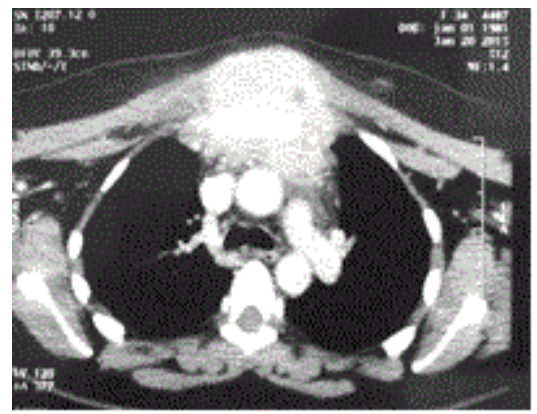

$1(\mathrm{~A})$

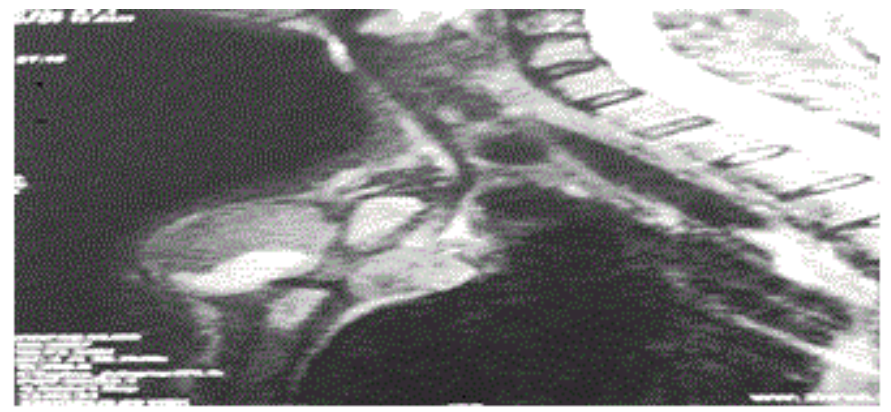

$1(\mathrm{~B})$

Figure 1: (A) CT-scan and (B) MRI showed a $10 \mathrm{~cm}$ sternal mass centered by the manubriosternal articulation infiltrating left and right second sternocostal joints and intercostal muscles, with a contact with the aorta cross and the left brachiocephalic veins without an encroachment signs.

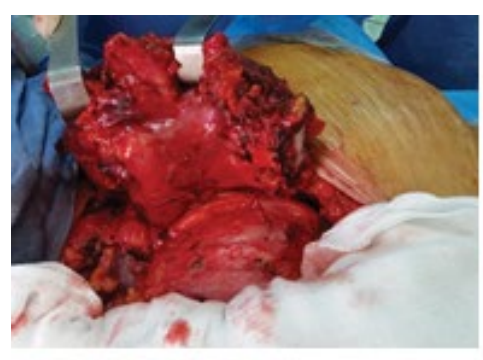

2(A)

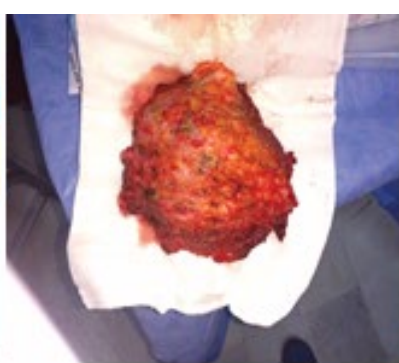

2(B)

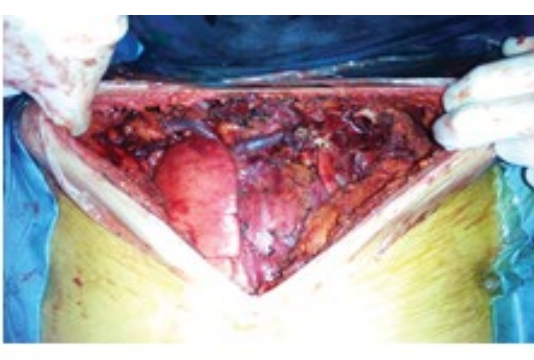

$2(\mathrm{C})$

Figure 2: (A) Resection of anterior arc of right ribs (from the first to the third), resection of internal right clavicle edge, transverse sternotomy above xiphoid process, resection of anterior arc of left ribs (from the first to the third), resection of internal left clavicle edge, (B) Sternal tumor and sternal body were removed en-bloc without the $2 \mathrm{~cm}$ residual extension which was marked by metallic clips, (C) Large anterior thoracic wall defect exposing mediastinum and medial portions of lungs.

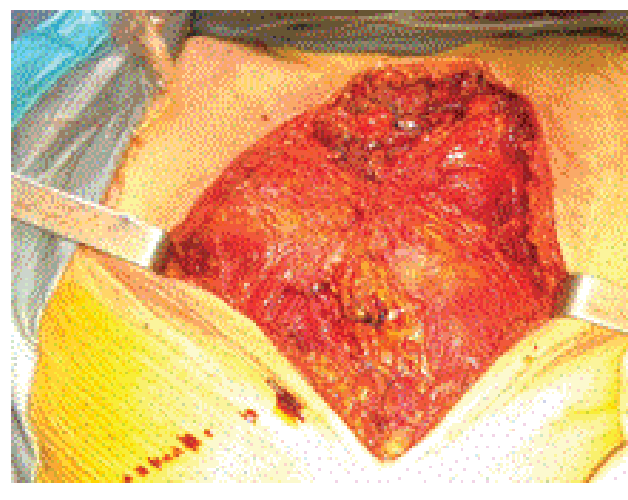

$3(\mathrm{~A})$

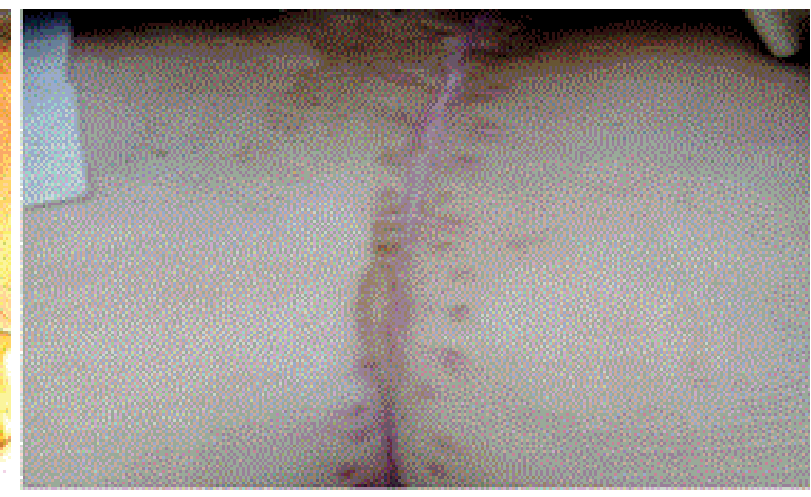

$3(\mathrm{~B})$

Figure 3: (A) Myoplasty using pectoralis major and pectoralis minor muscles covered the chest wall defect, (B) Surgery scar 3 months after the operation.

\section{Discussion}

\section{Indication and surgical resection}

Skeletal metastases of NPC occur in ribs and sternum in $7.8 \%$ of all skeletal metastases. Ma et al showed the impact of surgery, associated or not to chemotherapy, in solitary lung UCNT metastasis compared to radiotherapy or chemotherapy alone. The resection increased survival rate to $60.7 \%$ at 3 years (versus $56 \%$ with radiotherapy), the mean progression-free survival to 80.5 months (versus 37.8 months with radiotherapy) and the mean overall survival to 82.4 months (versus 49.6 months with radiotherapy). But solitary skeletal or sternal UCNT metastasis survival after surgery is not studied in the literature. In our case, the patient survived up to 24 months with a good quality of life after surgical resection followed by radiotherapy.

\section{Reconstruction}

Pectoralis major flap was used the first time in 1968 by Hueston to repair a loss of sternal substance. By transposing the muscle, the 
skin can be easily closed without an important distortion of breasts. When a large bilateral pectoral flap is used, the release of the muscle from its humeral attachment allows gaining in length and allows a better coverage of the chest wall defect. Indeed, pectoralis major flap offers a coverage characterized by its simple and immediate technique, flexibility, rigidity (relatively), resistance to infections and with acceptable esthetic results. Paradoxical respiration decreased after the adherence between visceral pleura and muscle flap.

The chest wall reconstruction is indicated after large resections following metastatic or contiguous neoplasm, post radiotherapy necrosis, infectious process, trauma and congenital parietal defect. If there is a risk of contamination after tumor necrosis or radiation tissue necrosis the use of prosthetic materials is not advisedv. We have also the experience to avoid prosthetic materials after an $\mathrm{R} 2$ resection because of the risk of tumor graft. Therefore, myocutaneous plasty is a possible solution when the use of prosthetic materials is impossible due to peroperative considerations such as infected or contaminated field wich exposes to chronic infection, or temporary closing before a second intervention [3-5].

Other muscles can be used to repair the anterior chest wall defect: Latissmus Dorsi, Rectus and Serratus are the most commonly used.
A consolidation by a bone auto graft is an alternative to enhance the stability of the rib cage. Several prosthetic materials are actually available and can be used in this case such as meshes and plates.

\section{Conclusion}

The use of muscle flaps is a good alternative to repair parietal defects. The choice of the muscle depends on the location and the extent of the defect to be repaired. The knowledge of the anatomy of the muscles is essential to obtain a good quality flap.

\section{References}

1. Losken A, Thourani VH, Carlson GW, Jones GE, Culbertson JH, et al. (2004) Reconstructive algorithm for plastic surgery following extensive chest wall resection. Br J Plast Surg 57: 295-302.

2. Sham JS, Cheung YK, Chan FL, Choy D (1990) Nasopharyngeal carcinoma: Pattern of skeletal metastases. Br J Radiol 63: 202-205.

3. Ma J, Wen ZS, Lin P, Wang X, Xie FY (2010) The results and prognosis of different treatment modalities for solitary metastatic lung tumor from nasopharyngeal carcinoma: A retrospective study of 105 cases. Chin J Cancer 29: 787-795

4. Hwang $K$ (2016) The origins of deltopectoral flaps and the pectoralis major myocutaneous flap. J Craniofac Surg 27: 1845-1848.

5. Shields WT (2011) General thoracic surgery. 